\title{
Dendritic Cells Induce Specific Cytotoxic T Lymphocytes against Prostate Cancer TRAMP-C2 Cells Loaded with Freeze- thaw Antigen and PEP-3 Peptide
}

\author{
Xiao-Qi Liu ${ }^{1}$, Rong Jiang ${ }^{2}$, Si-Qi Li ${ }^{1}$, Jing Wang ${ }^{1}$, Fa-Ping Yi ${ }^{1 *}$
}

\begin{abstract}
Prostate cancer is the most common cancer in men. In this study, we investigated immune responses of cytotoxic T lymphocytes (CTLs) against TRAMP-C2 prostate cancer cells after activation by dendritic cells (DCs) loaded with TRAMP-C2 freeze-thaw antigen and/or PEP-3 peptide in vitro. Bone marrow-derived DC from the bone marrow of the C57BL/6 were induced to mature by using the cytokine of rhGM-CSF and rhIL-4, and loaded with either the freeze-thaw antigen or PEP-3 peptide or both of them. Maturation of DCs was detected by flow cytometry. The killing efficiency of the CTLs on TRAMP-C2 cells were detected by flow cytometry, CCK8, colony formation, transwell migration, and wound-healing assay. The levels of the IFN- $\gamma$, TNF- $\beta$ and IL-12 were measured by enzyme-linked immunosorbent assay (ELISA). Compared with the unloaded DCs, the loaded DCs had significantly increased expression of several phenotypes related to DC maturation. CTLs activated by DCs loaded with freeze-thaw antigen and PEP-3 peptide had more evident cytotoxicity against TRAMP-C2 cells in vitro. The secretion levels of IFN- $\gamma$, TNF- $\beta$ and IL-12, secreted by DCs loaded with antigen and PEP-3 and interaction with $\mathrm{T}$ cells, were higher than in the other groups. Our results suggest that the CTLs activated by DCs loaded with TRAMP-C2 freeze-thaw antigen and PEP-3 peptide exert a remarkable killing efficiency against TRAMP-C2 cells in vitro.
\end{abstract}

Keywords: Dendritic cells - prostate cancer - tramp-c2 - cytotoxic t lymphocyte - immunotherapy

Asian Pac J Cancer Prev, 16 (2), 571-578

\section{Introduction}

Prostate cancer is a type of cancer because of the disorderly abnormal growth of the prostate gland acinar cells (Roupret et al., 2011). Globally, it is the second most common cause of cancer and the fifth leading cause of cancer-related death in men. The incidence of prostate cancer has obviously geographical and racial differences (Jacques et al., 2010; Siegel et al., 2012; Timothy et al., 2013). With the deterioration of environment and the aged tendency of population, the incidence of prostate cancer in china is steadily increasing year by year (Yi et al., 2010; Guo et al., 2010; Long et al., 2010), and shows a trend of younger. It is estimated that the aging population of china will exceed 200 million in 2014, which will have a profound social and economic effects. Therefore, it is important to research the innovative treatments.

With the development of immunology and immunological microenvironment study in various cancers, especially of dendritic cells (DCs), it will provide a new idea for prostate cancer treatment. DCs are the professional antigen-presenting cells of the immune system and the only one who can be able to activate the resting T lymphocytes, and play an important role in anti- tumor immune responses at the stage of initiate, control and maintenance (McDonnell et al., 2010; Lorenzi et al., 2011; Tel et al., 2013).

Loading whole tumor cells lysate is the most common way to deliver antigens to DCs (Bolhassani et al., 2011). Currently, only a part of tumor of TAA or TSA is known, while in large tumors are not yet clear. Whole tumor cells freeze-thaw antigens contain the entire repertoire of tumor antigens, including yet undefined TAA and individual mutations. It makes less likely to avoid antigen loss variants escape (Chiang et al., 2010). Epidermal growth factor receptor (EGFR) is a $170 \mathrm{kDa}$ glycoprotein product of the proto-oncogene c-erb $\mathrm{B}$, wherein the epidermal growth factor receptor variant III (EGRFRVIII) is the most common mutant (Yiin et al., 2010). PEP-3, a 13-amino acid sequence (LEEKKGNYVVTDHC) that spans the EGFRvIII mutation, can specifically activate the specific T lymphocyte immunity by binding to MHC-I and then induce CTLs to play an active anti-tumor role (Furnari et al., 1996). The systemic vaccination with a PEP-3 peptide, potent anti-tumor activity was generated against s.c. and established intracerebral tumors (Heimberger et al., 2003). This PEP-3 peptide vaccination results in antibody responses that are specific to the EGFR and may be also 
responsible for the efficacious responses seen within the prostate cancer.

In this study, DCs were induced by the bone marrowderived mononuclear cells, loaded with freeze-thaw antigen of whole tumor cells and/or PEP-3 peptide, and then co-cultured with allogeneic $\mathrm{T}$ lymphocytes to investigate whether the CTLs activated by loaded DCs has effective cytotoxicity to against prostate cancer TRAMP-C2 cells in vitro.

\section{Materials and Methods}

\section{Reagents}

PEP-3, a 13-amino acid peptide with a terminal cysteine (LEEKKGNYVVTDHC), was synthesized by Shanghai Science Peptide Biological Technology Co., LTD. The purity of the peptide preparations was more than $95 \%$, as assessed by high-pressure liquid chromatography. Recombinant murine IL-4 and GMCSF were purchased from Peprotech (USA). Antibodies of CD11c-FITC, CD80-PE, CD86-PE, CD40-PE and major histocompatibility complex-II-PE (MHC-II (IA/ IE)-PE) were purchased from eBioscience (San Diego, CA, USA). ELISA kits for IFN- $\gamma$, TNF- $\beta$ and IL- 12 were obtained from Shanghai Source Biological Technology co., LTD. Cell counting kit-8 (CCK-8) was purchased from Beyotime Institute of Biotechnology (Shanghai, China). Mouse Spleen Ficoll-Hypaque solution was obtained from Hao Yang Biological Manufacture Co., LTD (Tianjin, China). RPMI-1640 medium and fetal bovine serum (FBS) was purchased from Hyclone (Logan, UT, USA).

\section{Cell line and mice}

TRAMP-C2 cells (Shanghai Ann Commerce Co., LTD) were grown in RPMI 1640 supplemented with $10 \%$ fetal bovine serum, $2.5 \mathrm{~g} / \mathrm{L}$ anhydrous glucose, $1.5 \mathrm{~g} / \mathrm{L}$ $\mathrm{Na} 2 \mathrm{CO} 3$ and $0.11 \mathrm{~g} / \mathrm{L}$ sodium pyruvate at $37^{\circ} \mathrm{C}$ in $5 \%$ $\mathrm{CO}_{2}$. C57BL/6 (male, 5-6 week-old, weight average from $18 \mathrm{~g}$ to $22 \mathrm{~g}$ ) were provided by Laboratory Animal Center of Chongqing Medical University. All animal studies were approved by the Institutional Animal Care and Use Committee of the Chongqing Medical University.

\section{Preparation of the TRAMP-C2 freeze-thaw antigen and PEP-3 peptide}

The TRAMP-C2 cells in the logarithmic phase were harvested and washed 3 times with PBS, then re-suspended in normal saline $\left(1 \times 10^{7} \mathrm{cells} / \mathrm{ml}\right)$. The TRAMP-C2 were subsequently treated with three rapid freeze/thaw cycles, one cycle consists of 10 minutes in liquid nitrogen followed by $10 \mathrm{~min}$ at $37^{\circ} \mathrm{C}$ to melt entirely. The supernatants were collected after hypothermia super centrifuge 20 minutes $\left(4^{\circ} \mathrm{C}, 12000 \mathrm{rpm}\right)$, and filtered by $0.22 \mu \mathrm{m}$ microporous membrane filter. Then, the TRAMP-C2 freeze-thaw antigens were collected.

PEP-3 peptide was dissolved and diluted with PBS to a final concentration of $1 \mathrm{mg} / \mathrm{ml}$.

\section{DC generation from bone marrow}

Immature DCs were generated from C57BL/6 murine bone marrow as previously described (Inaba et al., 1992).
Briefly, bone marrow was extracted from the femurs and tibias of mice under sterile conditions. Erythrocytes were lysed with ammonium chloride. Cells were washed with serum free RPMI 1640 and cultured in 6-well plates containing RPMI 1640 medium supplemented with $10 \%$ (v/v) fetal bovine serum, 10ng/ml rhGM-CSF and rhIL-4. On days 3 and 5, half of the media were refreshed and fresh cytokine media were added. On days 7 , cells were obtained as immature bone marrow-derived DCs. The morphology of immature DCs was confirmed by microscope on days 3,5 and 7 .

\section{DC activation and flow cytometry analysis}

The immature DCs were collected and cultured in fresh half-medium $\left(1 \times 10^{6}\right.$ cells $\left./ \mathrm{ml}\right)$ containing freeze-thaw antigen (DC: TRAMP-C2 $=1: 10)$, PEP-3 peptide $(50 \mu \mathrm{g} /$ $\mathrm{ml}$ ), freeze-thaw antigen (DC: TRAMP-C2=1:5) and PEP3 peptide $(25 \mu \mathrm{g} / \mathrm{ml})$, respectively. The medium was used as a control. In brief, the different treatments were named as DC-antigen group, DC-PEP-3 group, DC-antigenPEP-3 group and DC-medium group, respectively. Each group was performed three times. The DCs were continued to incubate for $24 \mathrm{~h}$ at $37^{\circ} \mathrm{C}$ in $5 \% \mathrm{CO}_{2} . \mathrm{CD} 11 \mathrm{c}$ was used as a relative specific marker of DCs, while CD80, CD86, CD40 and MHCII were used to detect the maturation of DCs. The maturation analyses of DCs were performed using a FACS.

\section{Preparation of CTLs}

T-lymphocytes were isolated from the mouse spleen through the Ficoll-Hypaque density gradient centrifugation. Specific operation was performed according to the manufacturer's instructions. The four DCs groups above were washed twice and co-cultured with T-lymphocytes respectively for $24 \mathrm{~h}$ at the ratio of $1: 10$ to induce specific CTLs. Then, CTLs were co-cultured with TRAMP-C2 cells for $24 \mathrm{~h}$ and the cancer cell was taken as target cells at the ratio of effector to target of 10:1. All TRAMP-C2 cells were harvested and used for following experiments. The untreated TRAMP-C2 cells were used as the blank group.

\section{CCK-8 assay}

The TRAMP-C2 cells treated with CTLs were harvested and $2 \times 10^{3}$ cells/well were seeded in 96-well plates. The cells were maintained in $10 \%$ FBS RPMI 1640 medium at $37^{\circ} \mathrm{C}$ in $5 \% \mathrm{CO}_{2}$ for $24 \mathrm{~h}, 48 \mathrm{~h}, 72 \mathrm{~h}$, and $96 \mathrm{~h}$, respectively. $10 \mu \mathrm{l} \mathrm{CCK}-8$ solution were added into the cells and incubated for $1 \mathrm{~h}$ at $37^{\circ} \mathrm{C}$. A450 was detected by microplate reader, and the cell killing activity was calculated.

\section{Colony formation assay}

After treated with CTLs, the TRAMP-C2 cells in the logarithmic phase were dissociated into by $0.25 \%$ trypsin. The cells were resuspended in $2 \times$ RPMI 1640 culture medium. For each group, $3 \mathrm{ml} 45^{\circ} \mathrm{C}$ pre-warmed broth containing $10 \%$ FBS, $1 \%$ antibiotic medium and $0.6 \%$ sepharose solution were added into the bottom of the dish, then 4000 cells were seeded in dish with $2 \mathrm{ml}$ broth containing $10 \%$ FBS, $1 \%$ antibiotic medium and 
Dendritic Cells Induce the Specific Cytotoxic T Lymphocytes against TRAMP-C2 Prostate Cancer Cells

$0.35 \%$ sepharose solution. Cells were cultured at $37^{\circ} \mathrm{C}$ in $5 \% \mathrm{CO}_{2}$ and saturation humidity. When there were some macroscopic clones in the dishes emerging, the culture was terminated. Clones with more than ten cells were counted in four randomly selected visual fields from the central and peripheral portions of the wells at an inverted microscope.

\section{Apoptosis analysis}

The five groups of TRAMP-C2 cells in the up step were harvested and dissociated with $0.25 \%$ trypsin. The cells were resuspended in Annexin V-FITC binding buffer, and incubated at room temperature for $10 \mathrm{~min}$ in the dark. After centrifugation, cells were resuspended and PI staining solution was added (ABGAB, USA). Cell apoptosis were measured by flow cytometry.

\section{Cell migration assay}

The TRAMP-C2 cells were collected, washed, and resuspended in serum-free RPMI 1640 , and $2 \times 10^{4}$ cells were seeded on top of chamber of the transwell filters (Millipore, USA). 500 $\mu$ l RPMI 1640 medium containing $15 \%$ FBS were placed in the well below served as the attractant. The cells were allowed to migrate for $16 \mathrm{~h}$ at $37^{\circ} \mathrm{C}$ in a humidified incubator with $5 \% \mathrm{CO}_{2}$. Noninvasive cells were removed mechanically with a cotton tip from the upper surface of the membrane. Moreover, the invasive cells attached to the lower surface were fixed with methanol, stained with crystal violet for $20 \mathrm{~min}$, visualized and photographed with inverted microscope at $200 \times$ magnifications. Pictures of three random visual fields from three replicate wells were obtained and the number of migrated cells was counted.

\section{Wound healing assay}

Etching lines were drawn lightly on the bottom of the 6-well plates provided a good reference under the microscope. $5 \times 10^{5}$ TRAMP-C 2 cells/well were seeded into 6-well plate for overnight, the monolayer cells were scraped with a $10 \mu$ pipette tip and washed twice with PBS. Then cells were incubated with Serum-free medium for $24 \mathrm{~h}$, visualized and photographed with inverted microscope. The migrations of the cells can be determined by the reference point at time zero.

Enzyme-linked immunosorbent assay (ELISA) for IFN- $\gamma$, $T N F-\beta$ and $I L-12$

The supernatants were collected; IFN- $\gamma$, TNF- $\beta$ and
IL-12 concentrations in each group were measured by standard ELISA kits in triplicate. The tests were performed according to the manufacturer's instructions.

\section{Statistical analysis}

All statistical analyses were performed with the SPSS 22.0 statistical software package (SPSS Inc., Chicago, IL, USA). All the data were expressed as mean \pm SD. The significance was determined by the one-way analysis of variance (ANOVA) and LSD test. $p<0.05$ was considered statistically significant.

\section{Results}

Morphology of DCs generated from C57BL/6 bone marrow

The immature DCs were cultured in RPMI 1640 medium supplemented with the appropriate cytokine cocktail. On days 3, the shape of the adherent cells was looked like bean sprouts, and then branch-like protrusions were gradually significantly appeared (Figure 1A). On days 5, some suspension clusters cells were emerged (Figure 1B). On days 7, suspension cells were gradually increased and there were showed typical morphology such as large and branching structure aggregating among the cells (Figure 1C).

\section{Phenotypic characters of immature and mature DCs}

The immature DCs were stimulated to mature by loading the freeze-thaw antigen and/or PEP-3 peptide. Phenotypes of DCs were detected by flow cytomety to identify the immature and mature DCs. In immature DCs, CD11c, CD80, CD86, CD40 and MHC-II was $6.38 \%, 15.56 \%, 35.72 \%, 28.06 \%$ and $28.85 \%$ (Figure $2 \mathrm{~A})$, respectively.

However, these cell surface markers were significantly expressed in the mature DCs $(p<0.05$, Figure $2 \mathrm{~B})$, far more than the immature group (Figure $2 \mathrm{C}$ ), while there was no significant difference between the different types of mature DCs.

\section{CTLs inhibited proliferation and promoted apoptosis in TRAMP-C2}

Colony formation and CCK8 assays were performed in TRAMP-C2 cells to evaluate the effect of DCs loaded with freeze-thaw antigen and/or PEP-3 peptide. Colony formation rate reflects two important traits including the

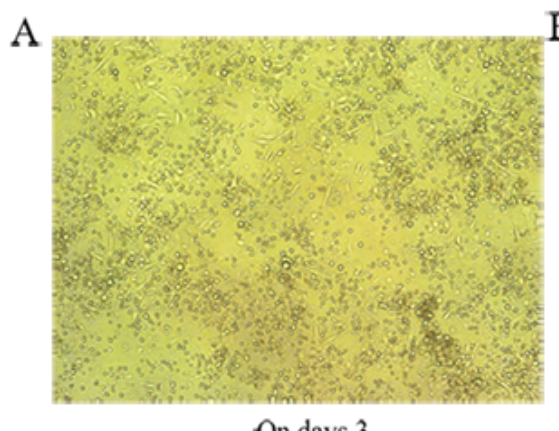

On days 3
B

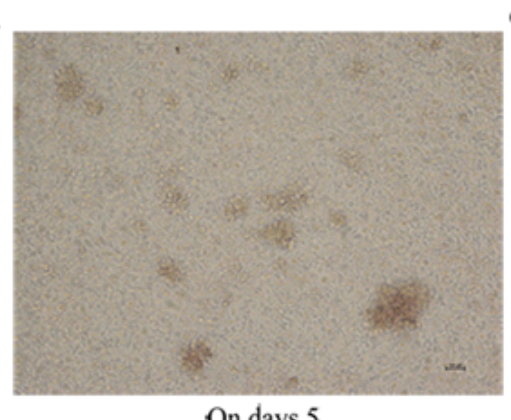

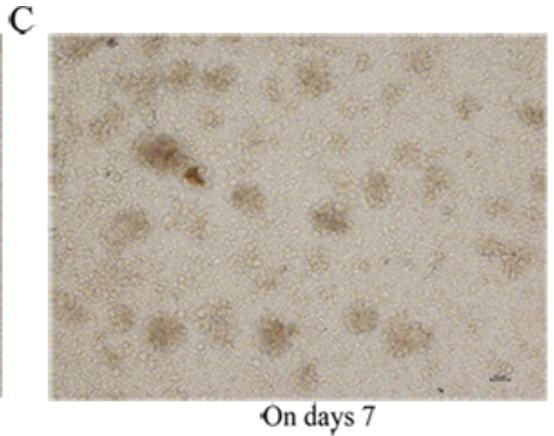

Figure 1. The Morphology of Immature Dendritic Cells Observed Under a Microscope (×100) on days 3(A), 5(B) and 7(C), respectively. 
A
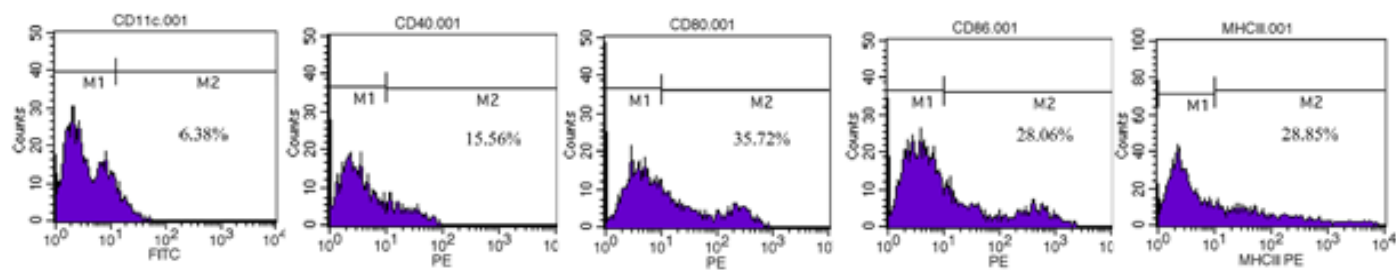

B
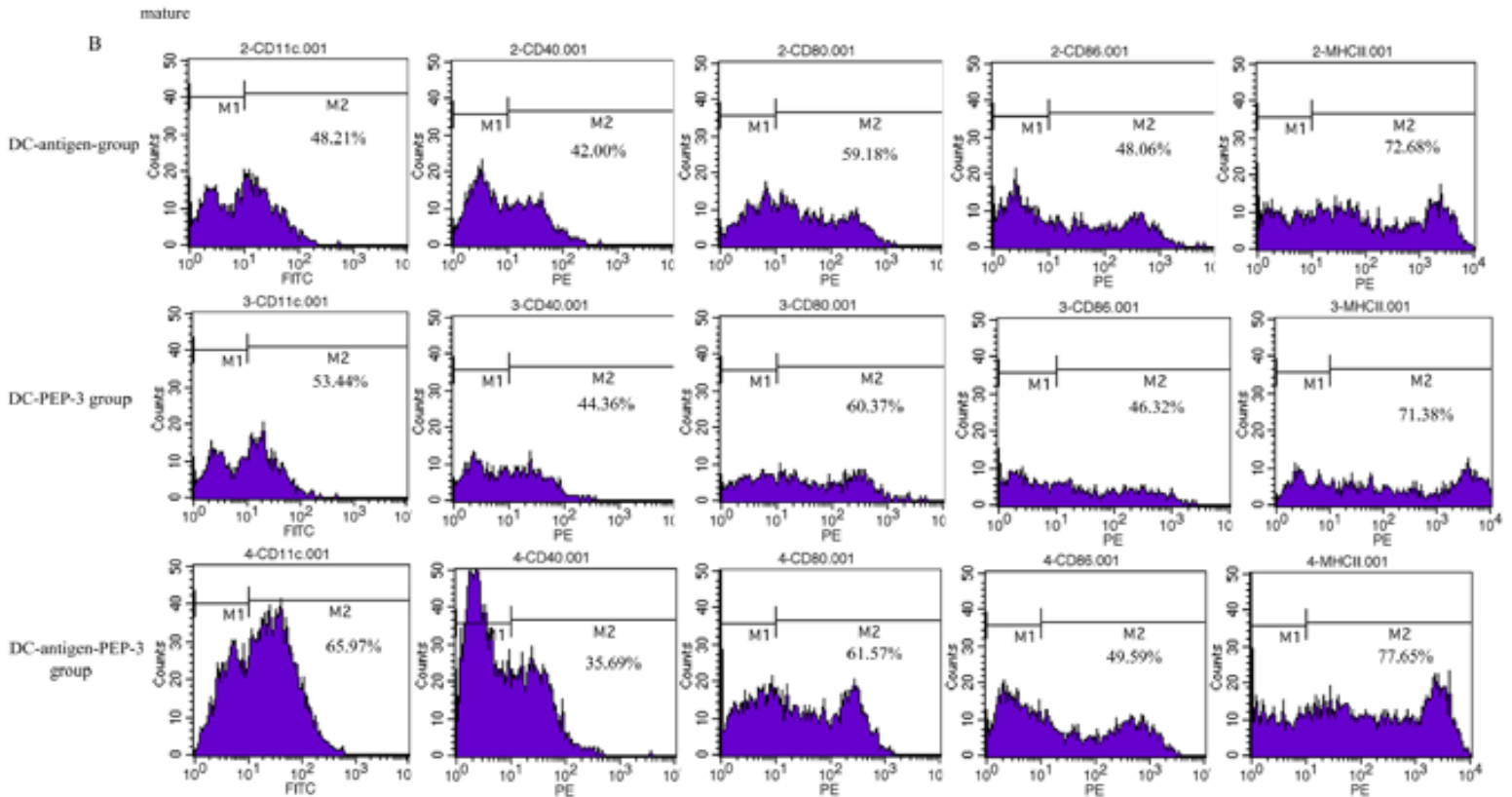

C

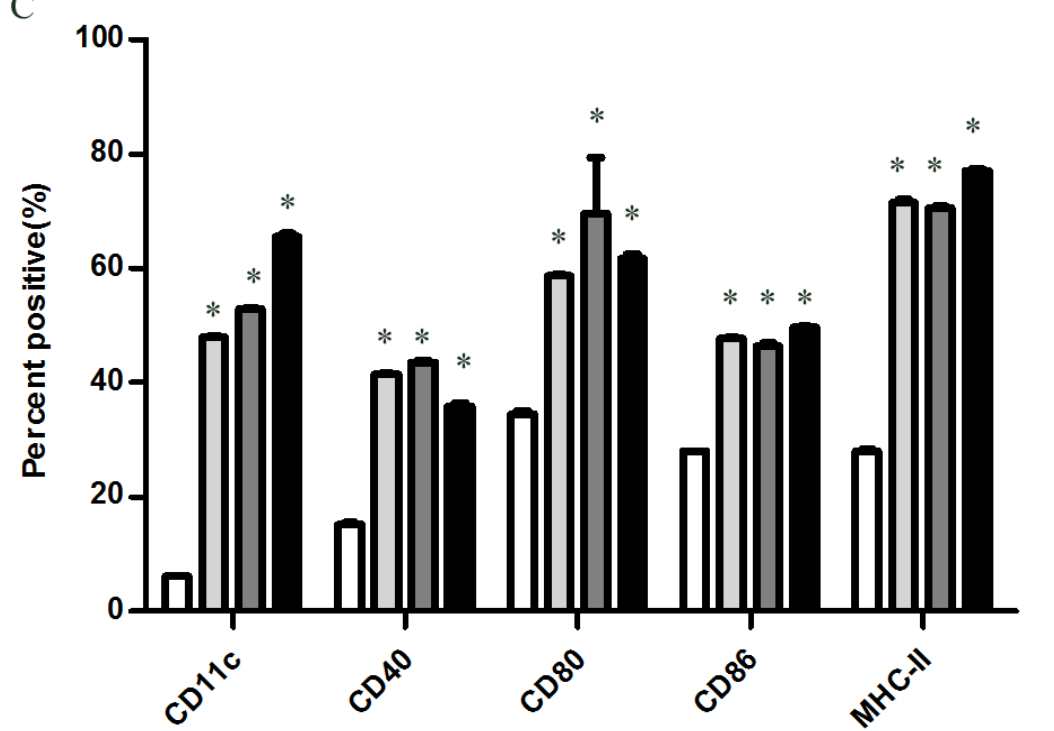

immature

DC-antigen group

DC-PEP-3 group

DC-antigen-PEP-3 group

Figure 2. The Phenotypes of Immature and Mature DCs. (A): Expressions of CD11c, CD40, CD80, CD86 and MHC-II in immature DCs. (B): Expressions of CD11c, CD40, CD80, CD86 and MHC-II in mature DCs. The expression of these phenotypes in mature DCs is higher than in immature DCs, but there was no significant difference between the mature DCs. (C): The positive percent $(\%)$ of the immature and mature DCs

cell group dependence and proliferation ability. In the colony formation assay, the colony formation efficiency of the treated group cells were lower than that of the blank and NC group (DC-medium group) cells $(p<0.05)$ (Figure $3 \mathrm{~A})$. The colony-forming number of the TRAMP-C2 cells (DC-antigen-PEP-3-C2) which were co-cultured with CTLs induced by DCs loaded with antigen and PEP-3 was far lower than that of the DC-antigen group (DC-antigen-C2) and DC-PEP-3 group (DC-PEP-3-C2) $(p<0.05$, Figure $3 \mathrm{~B})$, while there was no significant difference between the latter two groups. Furthermore, CCK8 assay were performed in order to study the effect of CTLs on cell proliferation. The proliferation ability of the treated group cells was decreased from $24 \mathrm{~h}$, while compared with the blank and NC groups. Moreover, the lowest group was the DC-antigen-PEP-3-C2 group $(p<0.05)$, and there was no significant difference between the DC-antigen-C2 and DC-PEP-3-C2 group (Figure 3C).

To further explore the apoptosis induced by DCs, the TRAMP-C2 cells were stained with Annexin-V/PI and 
Dendritic Cells Induce the Specific Cytotoxic T Lymphocytes against TRAMP-C2 Prostate Cancer Cells

analyzed by flow cytometry. The results showed that the treated groups had more significant apoptosis rate, whereas the rate could reach up to $14.02 \%$ in DC-antigen-PEP-3 group, more significantly than other groups $(p<0.05)$ (Figure 3D).

CTLs inhibited migration and invasion in TRAMP-C2

Transwell and wound-healing assay were performed in TRAMP-C2 cells to evaluate the migratory and invasion activity. The number of migrated cells in treated groups was less than that of the control groups (Figure 4A). Moreover, it was significant lower in DC-antigenPEP-3-C2 group, and there had no significant difference between the DC-antigen-C2 and DC-PEP-3-C2 groups $(p<0.05$, Figure 4B). Additionally, the migration cells in wound-healing assay could be determined by the number
A
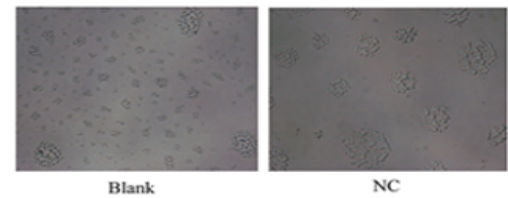

$\mathrm{NC}$
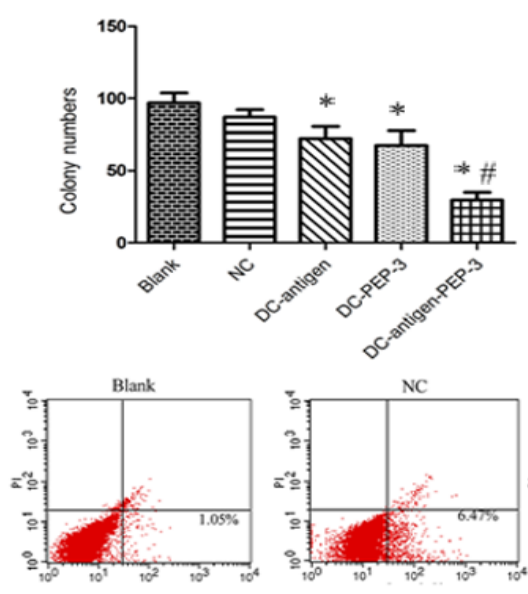

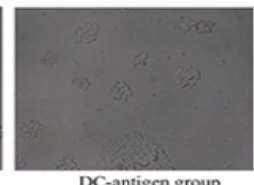

c.

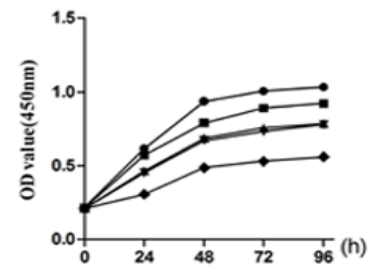

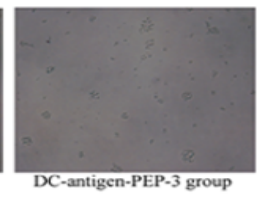

$\rightarrow$ Blank

$-\mathrm{NC}$

+ DC-antigen group

$\rightarrow$ DC-PEP-3 group

$\rightarrow$ DC-antigen-PEP-3 group

Figure 3. The Proliferation and Apoptosis of TRAMP-C2 cells were Detected as Follows. Blank, normal cultured TRAMP-C2 cells; NC, the DC-medium induced CTLs treated TRAMP-C2 cells. (A): The colony formation efficiency of the five groups of TRAMP-C2 cells. (B): A significant reduction in the number of colonies in TRAMP-C2 cells was observed in the loaded groups as compared with the blank and $\mathrm{NC}$ groups $(p<0.05)$, in which the DC-antigen-PEP-3 group has the lowest colony numbers $(p<0.05) .{ }^{*} p<0.05$ vs. the blank and NC groups; $\# p<0.05$ vs. the DC-antigen and DC-PEP-3 groups. (C): CCK 8 assay was performed in five groups of TRAMP-C2 cells. Loading of freeze-thaw antigen and/or PEP-3 peptide had significant inhibitory effects on the proliferation of TRAMP-C2 cells as compared with the blank and NC groups $(p<0.05)$. Significant proliferation decreases in loaded groups were observed in the DC-antigen-PEP-3-C2 group $(p<0.05)$. (D): Flow cytometry analysis of apoptosis of TRAMP-C 2 cells. The killing efficiency of the loaded DCs induced CTLs was obviously stronger than the blank and NC groups, while the DC-antigenPEP-3 induced CTLs was higher than the DC-antigen and DC-PEP-3 induced CTLs
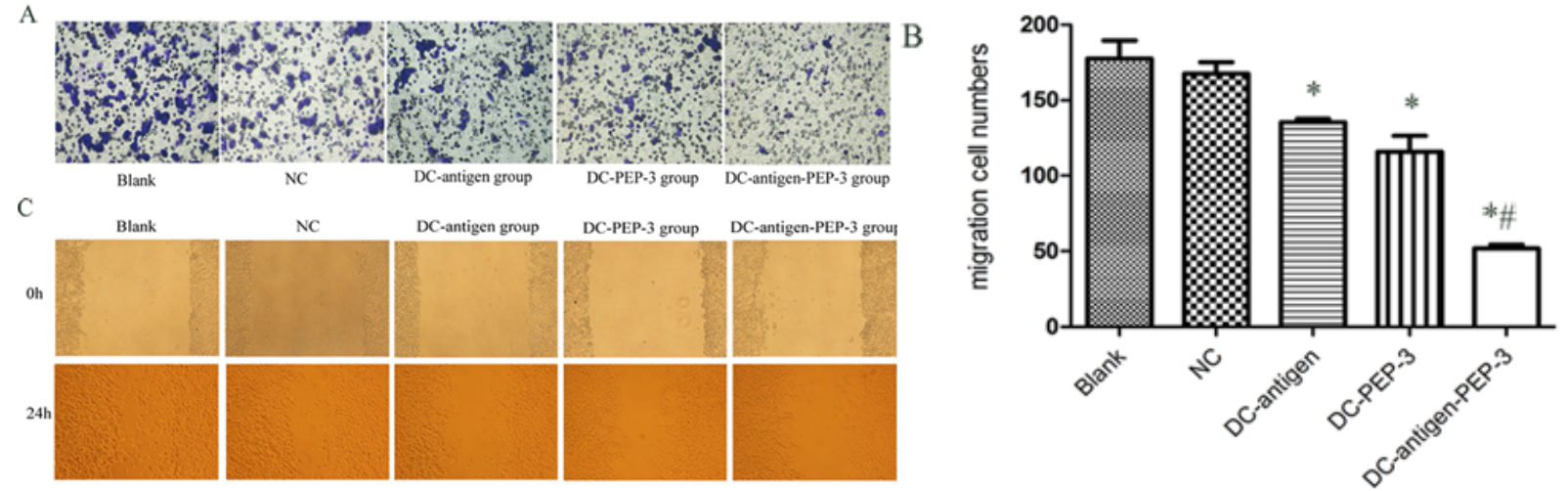

Figure 4. The Invasive and Migratory Ability of TRAMP-C2 Cells was Assessed by Transwell Assay and WoundHealing Assay. Blank, normal cultured TRAMP-C2 cells; NC, the DC-medium induced CTLs treated TRAMP-C2 cells. A): The TRAMP-C2 cells penetrating the membrane was lower in the loaded group than in either the blank or NC groups $(p<0.05)$. B): The ability of invasion is expressed as the number of cells which have migrated through the membrane. Each one represents a mean value of three random visual fields from three replicate wells in each group. The DC-antigen-PEP-3-C2 group is the lowest $(p<0.05)$. ${ }^{*} p<0.05$ vs. the blank group and NC groups; ${ }^{*} p<0.05$ vs. the DC-antigen and DC-PEP-3 groups. C): Both the blank and NC groups contained a higher density of cells at $24 \mathrm{~h}$ after wounding, as compared with the loaded groups. The DC-antigen-PEP-3-C2 group has a lower density of cells in loaded groups 

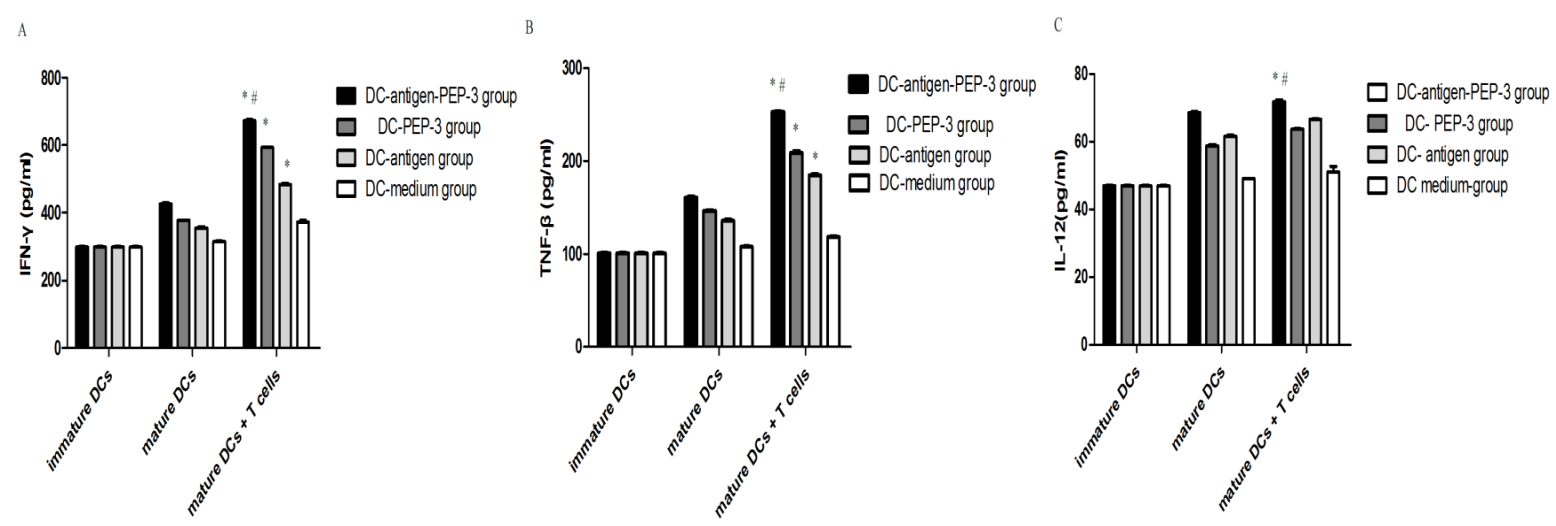

Figure 5. The Levels of IFN- $\gamma$ (A), TNF- $\beta$ (B) and IL-12 (C) Secreted by Immature DCs, Mature DCs and Mature DCs+T Cells. The mature DCs+T cells group showed a higher level as compared to the immature and mature DCs groups, in which the DC-antigen-PEP-3 group was higher than the DC-antigen and DC-PEP-3 group $(p<0.05) .{ }^{*} p<0.05$ vs. the immature and mature DCs groups; ${ }^{*} p<0.05$ vs. the DC-antigen and DC-PEP-3 groups

of cells that cross into the wound area from their reference point at time zero (Figure 4C). There had fewer cells in treated groups at $24 \mathrm{~h}$ post-wounding than in control groups. Moreover, the DC-antigen-PEP-3-C2 group had the fewest cells.

\section{High level of several cytokine secreted by DCs}

One of the best ways of determining the DCs function is to examine cytokine level. The secretion levels of IFN- $\gamma$, TNF- $\beta$ and IL-12 were measured by enzyme-linked immunosorbent assay for three replicate experiments. As a result, the secretion levels of IFN- $\gamma$ (Figure 5A), TNF- $\beta$ (Figure 5B) and IL-12 (Figure 5C) in mature DCs, which interaction with $\mathrm{T}$ cells, were higher than that in immature and mature DCs $(p<0.05)$, and the DC-antigen-PEP-3 group was higher than the DC-antigen and DC-PEP-3 group $(p<0.05)$. Furthermore, the IFN- $\gamma$, TNF- $\beta$ and IL12 secretion levels could reach $672.45 \mathrm{pg} / \mathrm{ml}, 252.71 \mathrm{pg} /$ $\mathrm{ml}$ and $71.92 \mathrm{pg} / \mathrm{ml}$, respectively.

\section{Discussion}

A complete understanding of the causes of prostate cancer remains elusive. The primary risk factors are obesity, age and family history, and most patients have locally advanced or metastasis when their first visit. Treatments of cancer have conventionally consisted of surgery, radiation and chemotherapy. Despite improved cancer therapy involving early detection, the surgical removal of solid tumor masses, and the use of radiotherapy, cytotoxic agents, or both, truly effective therapeutic approaches are still lacking and thus urgently needed (Matera et al., 2011).

In the modern era of prostate cancer treatment, rapid progress has led to a mass of therapeutic strategies for improving clinical outcomes. Specific targeting of therapeutic agents to tumors, with minimization of systemic toxicity, has been a goal of cancer therapy for more than a century. With the development of the immunology, cancer immunotherapy has been increasingly attracted our attention. There are three main groups of immunotherapy used to treat cancer: cell-based therapies, antibody therapies and cytokine therapies. Cell- based therapies , also known as cancer vaccines, contain four cell types that are natural killer cells, lymphokineactivated killer cells, cytotoxic T cells and dendritic cells, while the dendritic cells are the most commonly used cells. It has been used in many malignancies, and the most commonly seen is in prostate cancer, melanoma, colorectal carcinoma and multiple myeloma (Drake et al., 2010; Yang et al., 2010; Lee et al., 2012).

DCs have been demonstrated to have the exceptional ability to effectively uptake, process and present antigen to activate naive CD4+ and CD8+ T cells and initiate the antigen-specific antibody synthesis. By high expression of the major histocompatibility complex Iand II on the cell surface, DCs presented the tumor antigen peptide and fully occupied the relevant T lymphocyte receptor. At the same time, DCs provided the high level of co- stimulatory molecules CD80, CD86 and CD40 to activate the T cells. Moreover, DCs also play an anti-tumor role by synthesis and secretion of cytokines, such as IL-12 (Morandi et al., 2006).

DC-based vaccinations have been used in clinical studies to induce tumor-specific immunity as well as clinical responses in selected patients. Strategies to load tumor antigens into DCs have included loading with defined tumor antigens (peptides or proteins, transfer of tumor antigen-specific DNA or RNA) (Kreiter et al., 2011; Kang et al., 2013) or whole tumor cell lysates (Chiang et al., 2011). It has been shown that peptide-loaded DCs increase the peptide-specific cytotoxic T-lymphocytes in melanoma, renal cell carinoma, breast and ovarian carcinoma patients (Quintarelli et al., 2011; Clive et al., 2012). However, peptide-based vaccines are limited in their application, as they are restricted to the selected peptides and will only be effective for patients that express enough HLA, which presents these particular antigenic peptides for recognition by the $\mathrm{T}$ lymphocytes. In contrast to peptide loading, using whole tumor cells freeze-thaw antigen for DC-loading has the advantage that the entire repertoire of tumor antigens, including yet undefined TAA and individual mutations, are presented, making it less likely that antigen loss variants would escape (Park et al., 2011). Gilboa et al. (1988) also suggests that effective vaccine formulations should contain mixtures of 
tumor antigens. EGFRvIII is the most common mutation of EGFR. It is characterized by a consistent in-frame deletion of 801 base pairs from the extracellular domain, which splits a codon and produces a novel glycine amino acid at the fusion junction. This fusion junction encodes a tumor-specific protein sequence that is expressed on the surface of tumor cells but is not present in normal tissues (Hong et al., 2002), making it an ideal target for anti-tumor immunotherapy, while PEP-3 is the most commonly used peptide of the EGFRvIII vaccine. Our study is also strongly suggested that the combination use of tumor whole tumor cells freeze-thaw antigen and PEP-3 peptide could enormously improve the killing efficiency of CTLs. Hence, loading with mixtures of tumor-derived antigens and peptide may be a better way.

Mature DCs can highly express MHC and costimulatory molecules and activate the $\mathrm{T}$ cells to initiate the immune response. After loading of freeze-thaw antigen and/or PEP-3 peptide, the expression of MHC and co-stimulatory molecules were significantly increased. This may suggest that freeze-thaw antigen and/or PEP-3 peptide can be used as a stimulus signal to up-regulation the expression of MHC and co-stimulatory molecules, so it promotes the maturation of DCs.

Proliferation and apoptosis are two important features of cell. Tumor cell proliferation and apoptosis are closely related to the biological behaviors of growth, invasion, recurrence, metastasis and prognosis. In this study, the killing efficiency of CTLs on TRAMP-C 2 was detected by colony formation, CCK 8 assay, flow cytometry, transwell assay and wound-healing assay. After co-cultured with CTLs induced by DCs loading of freeze-thaw antigen and/ or PEP-3 peptide, the proliferation ability of TRAMP-C2 was inhibited and the apoptosis was increased when compared with the control groups. Furthermore, the CTLs induced by DCs both loaded of freeze-thaw antigen and PEP-3 peptide has the lowest proliferation and the highest apoptosis. These results may suggest that freeze-thaw antigen and PEP-3 peptide could promote the maturation of DCs and make the activated CTLs have more evident killing efficiency on TRAMP-C2 in vitro.

Dendritic cells secrete chemokines that selectively attract naive and mature T cells (Stockwin et al., 2000). Recognition of peptide-MHC complexes on DC by antigen-specific $\mathrm{T}$ cell receptors (TCR) then initiates DC-T-cell interaction. Interaction between co-stimulatory molecules on DCs and their ligands on T cells are required to sustain and amplify T-cell activation. Activated CD4+ $\mathrm{T}$ cells can in turn activate DC to release high levels of IFN- $\gamma$, TNF- $\beta$ and IL- 12 . These cytokines can induce the $\mathrm{T}$ cell and NK cells to produce large amounts of tumor necrosis factor, perforin and granzyme to make the tumor cells dissolved. The secretion levels of IFN- $\gamma$, TNF- $\beta$ and IL-12 in loaded groups were higher than the unloaded group. After interaction with $\mathrm{T}$ cells, the secretion level of the cytokines was significantly increased. This may suggest that freeze-thaw antigen and/or PEP-3 peptides can be used as a stimulus signal to increase the cytokines secretion of DCs, and promote the function of DCs to maturation.

In summary, we have demonstrated the role of freeze- thaw antigen and/or PEP-3 peptides in DCs immune response. Our data revealed that loading of freeze-thaw antigen and PEP-3 peptides can significantly increased the anti-tumor capacity of DCs. This work may aid the development of new therapeutic strategies for prostate cancer treatment.

\section{Acknowledgements}

This research is supported by grant funding from Natural Nature Science Foundation of China (no. 30800945).

\section{References}

Bolhassani A, Safaiyan S, Rafati S (2011). Improvement of different vaccine delivery systems for cancer therapy. Mol Cancer, 10, 3 .

Chiang CL, Benencia F, Coukos G (2010). Whole tumor antigen vaccines. Semin Immunol, 22, 132-43.

Chiang CL, Hagemann AR, Leskowitz R, et al (2011). Day-4 myeloid dendritic cells pulsed with whole tumor lysate are highly immunogenic and elicit potent anti-tumor responses. PLoS One, 6, 28732.

Clive KS, Tyler JA, Clifton GT, et al (2012). The GP2 peptide: A HER2/neu-based breast cancer vaccine. J Surg Oncol, 105, 452-8.

Drake CG (2010). Prostate cancer as a model for tumor immunotherapy. Nat Rev Immunol, 10, 580-93.

Furnari FB, Huang HJ, Cavenee WK (1996). Molecular biology of malignant degeneration of astrocytoma. Pediatr Neurosurg, 24, 41-9.

Guo K, Liu CX (2010). Research progress on the serum markers of prostate cancer. J Practical Med, 26, 3465-6.

Gilboa E, Nair SK, Lyerly HK (1998). Immunotherapy of cancer with dendritic-cell-based vaccines. Immunother, 46, 82-7.

Hong Ge, Xiaoqi Gong, Careen K (2002). Evidence of high incidence of EGFRvIII expression and coexpression with EGFR in human invasive breast cancer by laser capture microdissection and immunohistochemical analysis. Int $J$ Cancer, 98, 357-61.

Heimberger AB, Crotty LE, Archer GE, et al (2003). Epidermal growth factor receptor variant VIII peptide vaccination is efficacious against established intracerebral tumors. Clin Cancer Res, 11, 4247-54.

Inaba K, Inaba M, Romani N, et al (1992). Generation of large numbers of dendritic cells from mouse bone marrow cultures supplemented with granulocyte/macrophage colonystimulating factor. $J$ Exp Med, 176, 1693-702 .

Jacques Ferlay, Hai-Rim Shin, Freddie Bray, et al (2010). Estimates of worldwide burden of cancer in 2008: GLOBOCAN 2008. Int J Cancer, 127, 2893-917.

Kreiter S, Diken M, Selmi A, et al (2011). Tumor vaccination using messenger RNA: prospects of a future therapy. Curr Opin Immunol, 23, 399-406.

Kang TH, Mao CP, La V, Chen A, et al (2013). Innovative DNA vaccine to break immune tolerance against tumor self-antigen. Hum Gene Ther, 24, 181-8.

Lee HJ, Hong CY, Kim MH, et al (2012). In vitro induction of anterior gradient-2-specific cytotoxic $\mathrm{T}$ lymphocytes by dendritic cells transduced with recombinant adenoviruses as a potential therapy for colorectal cancer. Exp Mol Med, 44, 60-7.

Lorenzi S, Mattei F, Sistigu A, et al (2011). Type I IFNs control antigen retention and survival of CD8a (+) dendritic cells after uptake of tumor apoptotic cells leading tocrosspriming. 
Xiao-Qi Liu et al

$J$ Immunol, 186, 5142-50.

Long JR, Xiong F, Dong ZQ (2010). Research progress on multiple drug resistance of prostate cancer. Guangdong Med J, 31, 2871-3.

McDonnell AM, Prosser AC, Van Bruggen I, et al (2010). CD8alpha+DC are not the sole subset cross-presenting cell-associated tumor antigens from a solid tumor. Eur $J$ Immunol, 40, 1617-27.

Matera L (2011). The choice of the antigen in the dendritic cell-based vaccine therapy for prostate cancer. Cancer Treat Rev, 36, 131-41.

Morandi F, Chiesa S, Bocca P, et al (2006). Tumor mRNAtransfected dendritic cells stimulate the generation of CTL that recognize neuroblastoma-associated antigens and kill tumor cells: immunotherapeutic implications. Neoplasia, 10, 833-42.

Park MH, Yang DH, Kim MH, et al (2011). Alpha-Type 1 polarized dendritic cells loaded with apoptotic allogeneic breast cancer cells can induce potent cytotoxic T lymphocytes against breast cancer. Cancer Res Treat, 43, 56-66.

Quintarelli C, Dotti G, Hasan ST, et al (2011). High-avidity cytotoxic-T-lymphocytes specific for a new preferentially expressed antigen of melanoma (PRAME)-derived peptide can target leukemic- and leukemic-precursor cells. Blood, 117, 3353-62.

Roupret M, Zigeuner R, Palou J, et al (2011). European guidelines for the diagnosis and management of upper urinary tract urothelial cell carcinomas: 2011 update. Eur Urol, 59, 584-94.

Siegel R, Naishadham D, Jemal A (2012). Cancer statistics. $C A$ Cancer J Clin, 62, 10-29.

Stockwin LH, McGonagle D, Martin IG, et al (2000). Dendritic cells immunological sentinels with a central role in health and disease. Immunol Cell Biol, 78, 91-102.

Timothy R, Susan S, Bao-Li Chang, et al (2013). Global patterns of prostate cancer incidence, aggressiveness, and mortality in men of African descent. Prostate Cancer, 2013, 1-12.

Tel J, Schreibelt G, Sittig SP, et al (2013). Human plasmacytoid dendritic cells efficiently cross-present exogenous Ags to CD8+ T cells despite lower Ag uptake than myeloid dendritic cell subsets. Blood, 121, 459-67.

Yi XM, Zhou WQ (2010). Epigenetics of prostate cancer. National J Androl, 16, 635-41.

Yiin JJ, Hu B, Schornack PA, Sengar RS, et al (2010). ZD6474, a multitargeted inhibitor for receptor tyrosine kinases, suppresses growth of gliomas expressing an epidermal growth factor receptor mutant, EGFRvIII, in the brain. $M o l$ Cancer Ther, 9, 929-41.

Yang DH, Kim MH, Hong CY, et al (2010). Alpha-type 1-polarized dendritic cells loaded with apoptotic allogeneic myeloma cell line induce strong CTL responses against autologous myeloma cells. Ann Hematol, 89, 795-801. 